\title{
Ultraviolet, Water, and Thermal Aging Studies of a Waterborne Polyurethane Elastomer- based High Reflectivity Coating
}

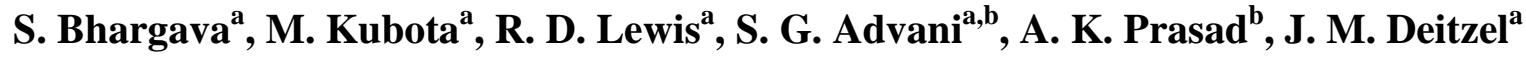 \\ ${ }^{\text {a }}$ Center for Composite Materials, University of Delaware, Newark \\ ${ }^{\mathrm{b}}$ Department of Mechanical Engineering, University of Delaware, Newark
}

\author{
Corresponding author: \\ Munetaka Kubota \\ Center for Composite Materials, University of Delaware \\ 202 Composites Manufacturing Science Laboratory \\ Newark, DE 19716, USA \\ Tel: 302-831-0272 \\ Fax: 302-831-8525 \\ Email: kubotam@udel.edu
}

\begin{abstract}
:
A waterborne aliphatic polyurethane-based coating was studied for accelerated ultra-violet (UV), water (WT), and thermal (TH) aging for a period of 1000 hours. To monitor the coating durability, samples were tested every 200 hours. ATR-FTIR spectroscopy was used to monitor the chemical changes occurring during the aging process. UV/Vis with integrating sphere was used to track the change in diffused reflectance, while the optical microscope and the scanning white light interferometry (SWLI) were used for surface characterization. FTIR studies of coatings subjected to UV exposure indicated a decrease in functional groups such as $\mathrm{CO}-\mathrm{NH}, \mathrm{C}-$ $\mathrm{H}, \mathrm{C}=\mathrm{O}$, and $\mathrm{C}-\mathrm{O}-\mathrm{C}$. The appearance of functional groups such as $\mathrm{N}-\mathrm{H}$ is attributed to chain scission of the polyurethane binder in the coating. Investigation of the degradation mechanism in water and thermal aging showed physical effects through water penetration and the mismatch in the coefficient of thermal expansion as the primary causes of degradation. In all aging scenarios, the reduction of reflectivity was largely due to physical defects caused by the different aging mechanisms.
\end{abstract}

Keywords: Waterborne coatings; Polyurethanes; UV-curing; Aging 


\section{Introduction}

As industries and consumers become more aware of their environmental impact, highreflectivity coatings have gained popularity for their energy-saving properties in applications such as lighting panels and reflective roof coatings [1,2]. With the recent tightening of EPA emissions regulations of volatile organic compounds (VOC) under the Clean Air Act [3], there is a movement towards non-solvent based systems for use in coatings and paints. In particular, water-based systems have become a popular non-solvent based option. Aqueous polyurethane dispersion coatings are an attractive choice due to their lack of VOCs, flexibility, and durability, along with their high adhesive strength [4]. It is noteworthy that solvent-based polyurethanes have been used in the coating industry for over 55 years [5].

Coatings are expected to be durable and retain their properties over time. For this reason, resistance to light, humidity and temperature, is a general requirement. One can further reduce the environmental impact of the coatings by increasing their efficiency and lifetime. The most common cause of coating degradation is the ambient exposure to ultraviolet (UV) radiation, water/humidity, and temperature fluctuation. In many cases, degradation is evaluated based on the changes in chemical structure and the presence of foreign chemicals in the system as a function of time.

Chemical evaluation of the degradation mechanisms often is conducted using attenuated total reflectance Fourier transform infrared (ATR-FTIR) spectroscopy [6, 8, 9, 10]. ATR-FTIR allows for the evaluation of chemical bonds present before and after aging. By evaluating the making and breaking of chemical bonds, one can gain insight into the degradation mechanisms present during the different aging processes. These chemical changes in the system can cause physical changes that can be observed using optical microscopy or SEM. In the case of coatings, degradation can also often be studied by monitoring color changes, using devices such as a spectrometer.

In this study, we prepared a high reflectivity water-based aliphatic polyurethane coating. We have simulated natural exposure to the coating by QUV irradiation, thermal cycling, and water immersion $[6,7]$. Previous studies have shown that UV radiation, heat, and high humidity have degrading effects on polyurethane materials [6]. ATR-FTIR was used to evaluate the resulting chemical changes. We then correlated the observed chemical changes to the performance degradation as a function of aging to understand the contribution of the chemical changes to the physical changes.

\section{Materials and Experimental Methods}

\subsection{Coating Formulation}

An aqueous polyurethane dispersion system (similar to that found in US Patent 8,734,940 B2 Example 12) [11] was used for the formulation of the system. This polyurethane system was partially cross-linked prior to coating the aluminum substrate. Due to the many different constituents and to ensure good pigment dispersion and break down pigment agglomerates, this 

stage.

\section{Titania Pre-grind Stage}

The titania pre-grind is a combination of distilled water, dispersing agent, defoarmer and $\mathrm{TiO}_{2}$ pigment. To create this portion of the system, distilled water and dispersing agent is mixed together. This is followed by the addition of the defoamer, followed by the $\mathrm{TiO}_{2}$ pigment. Using an angled-tooth blade or Cowles style propeller $(6.35 \mathrm{~cm}$ diameter $)$, the titania pre-grind is mixed under high shear speeds of between 2000-2400 rpm for 10 minutes to ensure complete dispersion and de-agglomeration of titania particles. To counter act the foam produced from the high shear mixing, the defoamer was added to the formulation.

The dispersing agent used in this system was a solution of a high molecular weight block copolymer with a large number of pigment affinic (anchor) groups which have a high affinity for pigment surfaces, which establish electrostatic repulsion between the particles as well as steric hindrance $[12,13,14]$. A ladder series test was conducted to determine the weight percentage of the dispersing agent needed. The dispersing agent was added in small amounts till the minimum viscosity was achieved. The defoamer used was a mixture of foam-destroying polysiloxanes and hydrophobic solids in polyglycol. The pigment used was a fine dry powder of rutile titania, with a median particle size of $0.41 \mu \mathrm{m}$.

\section{Let Down Stage}

The polyurethane binder, coalescing agent, and wetting and levelling agent were added to the Titania pre-grind prepared as discussed above in the let down stage. The formulation recipe of the system can be found in Table 1. The components are mixed using the Cowles blade at $1400 \mathrm{rpm}$ for 10 minutes and the formulation was set aside for 24 hours to allow for the defoamer to be fully effective

A waterborne aliphatic anionic polyurethane (PU) dispersion with a maximum of $2.5 \mu \mathrm{m}$ sized anionic particles was used as the binder. Waterborne coatings require coalescing agents to aid in good film formation. An ester-based coalescing agent was used to temporarily lower the $T_{g}$ and provide mobility to the polymer chains The lowering of the $\mathrm{T}_{\mathrm{g}}$ softens the polymer allowing for flow and coalescing of the polymer chains. Furthermore, this slows the drying process. To ensure good system to substrate wetting and leveling, an acrylic modified polyester (50 wt. \%) dissolved in the hydrophilic solvent dipropylene glycol (mono) methyl ether was added to the system.

\section{Table 1 Waterborne Paint Formulation Recipe}

\begin{tabular}{cc}
\hline Component & Wt.\% \\
\hline $\mathrm{TiO}_{2}$ & 62.85 \\
Polyurethane binder & 30.75 \\
Aliphatic poly(isocyanate) & 0.19 \\
cross-linker &
\end{tabular}


Polysiloxane defoamer $\quad 0.60^{\mathrm{a}}$

Coalescing agent 2.00

Wetting and leveling additive $\quad 0.84$

Distilled water $\quad$ Extra water added to keep constant \% of solvents

${ }^{\mathrm{a}} \mathrm{Wt}$ \% was determined by separate ladder study tests.

\section{Sample Preparation}

At the end of the 24 hours from the let down stage, a water-dispersible aliphatic poly(isocyanate) cross-linker (Easaqua XM-501) was added to the paint formulation at $1 \mathrm{wt} \%$ of solids weight of PU. This was immediately followed by slow shear mixing at 150-200 rpm for 5 minutes using the Cowles blade. The system was allowed to settle for 15 minutes prior to the draw-down of the coating. The draw-down was performed with a wire-wound rod (wet thickness $=6 \mathrm{mil}, 1 \mathrm{mil}=25.4$ microns) on a $0.041 \mathrm{~cm}$ thick $150 \mathrm{~mm}$ x $100 \mathrm{~mm}$ aluminum sheet. The coated substrate was cured and dried in an oven at $50{ }^{\circ} \mathrm{C}$ for 15 minutes. Samples $(100 \mathrm{~mm} \times 25$ $\mathrm{mm}$ ) were then cut using a specific shear method wherein two layers of breather cloth were placed on the sample catch. The sample was then covered with paper towels to prevent surface contamination.

\subsection{QUV Aging (UV)}

UV aging was performed using the QUV Accelerated Weathering Tester equipment from Q-Lab Corporation (Cleveland, USA) following the ASTM D 4674 [15] method (UV light cycle mode only, no condensation cycle). In the tester UVB-313 EL Fluorescent Lamps (Q-Lab Corporation, Ohio) with $0.7522 \mathrm{~W} / \mathrm{m}^{2} / \mathrm{nm}$ irradiance at $313 \mathrm{~nm}$ were used, with the panel temperature set at $50{ }^{\circ} \mathrm{C}$. The samples were subjected to aging for durations of $200,400,800$, and 1000 hours.

\subsection{Water Immersion Aging (WI)}

ASTM D 870-02 [16] was modified to test water resistance of coatings using water immersion. The samples were placed in $250 \mathrm{ml}$ Nalgene® bottles with three holes in the lid. The bottle was filled with deionized water such that the samples were fully immersed and placed in an oven at $38 \pm 1{ }^{\circ} \mathrm{C}$ in accordance to the ASTM D 870-02. The samples were removed every 200 hours and pat dried with paper towels for further evaluation.

\section{$2.5 \quad$ Thermal Aging (TH)}

An environmental chamber (ESPEC, ECT model) was used for freeze/thaw cycling. Table 2 lists the thermal cycling procedure used as per ASTM D 6944-03 [17]. The temperature of the chamber was ramped up from $-5{ }^{\circ} \mathrm{C}$ to $50{ }^{\circ} \mathrm{C}$ and back down again to $-5{ }^{\circ} \mathrm{C}$ during each cycle. For each cycle lasting 25 hours, the temperature was held at $-5{ }^{\circ} \mathrm{C}$ for 16 hours, 30 minutes to ramp to $50{ }^{\circ} \mathrm{C}$, then at $50{ }^{\circ} \mathrm{C}$ for 8 hours, another 30 minutes to ramp down to the original $-5{ }^{\circ} \mathrm{C}$, then this cycle is repeated. The sample was removed from the environmental 
chamber every five cycles and its performance was tested, and then returned to the chamber for another round of freeze/thaw cycling.

\begin{tabular}{cc}
\multicolumn{2}{c}{ Table 2 Thermal cycling procedure } \\
\hline Condition & Time (hours) \\
\hline $\begin{array}{c}-5 \pm 3^{\circ} \mathrm{C} \text { in air } \\
\text { Ramp Time from }-5^{\circ} \mathrm{C} \text { to } \\
50{ }^{\circ} \mathrm{C} \text { in air }\end{array}$ & 0.5 \\
$50 \pm 3{ }^{\circ} \mathrm{C}$ in air & 8 \\
Ramp Time from $-5^{\circ} \mathrm{C}$ to & 0.5 \\
$50{ }^{\circ} \mathrm{C}$ in air & \\
Repeat from beginning & 25 hours per cycle \\
\hline
\end{tabular}

\subsection{UV/Vis Spectrophotometry}

A single-beam Lambda 35 Perkin-Elmer UV-Vis spectrophotometer (Perkin-Elmer Corporation, USA) equipped with a Labsphere RSA-PE-20 integrating sphere attachment (Labsphere Inc., New Hampshire) was used to measure the optical diffused reflectance at room temperature. All measurements were taken using a $0^{\circ}$ sample holder to position the sample for illumination by the sample beam at $0^{\circ}$ incidence angle, i.e. normal incidence. $99 \%$ Spectralon ${ }^{\circledR}$ Diffuse Reflectance Standard (Labsphere Inc., New Hampshire) was used to calibrate the UVVis spectrophotometer. Samples were cut into $25 \mathrm{~mm}$ by $25 \mathrm{~mm}$ square samples and tested over the wavelength range of 380-750 nm, with a slit width of $2 \mathrm{~nm}$, and $240 \mathrm{~nm} / \mathrm{min}$ scan speed.

\subsection{Optical Microscope}

Optical microscope images were acquired using a Nikon Eclipse LV100 microscope with a Nikon Digital Sight DS-Fit camera (Nikon Corporation, Tokyo Japan). The images were taken through a $5 x$ objective lens and 10x eye piece.

\subsection{ATR-FTIR}

A Spotlight 400 Perkin Elmer FTIR Imaging System (Perkin Elmer Corp, Shelton, CT, USA) with wavenumber range of 750 to $4000 \mathrm{~cm}^{-1}$ was used to identify and quantify the chemical changes in the system. An Attenuated Total Reflectance (ATR) accessory was mounted on the microscope stage, and the sample was focused with the help of the microscope and ATR prism $(\mathrm{Ge})$ which was lowered until it contacted the sample. All scans including the background, FTIR spectra were recorded with a $100 \times 100 \mu \mathrm{m}$ aperture in the range of $750-1800 \mathrm{~cm}^{-1}$ with 2 $\mathrm{cm}^{-1}$ resolution. All final spectra were an average of 512 scans with the background subtracted. The Perkin Elmer Spotlight software (v.1.4.1) was used for recording and processing the ATRFTIR spectra.

\subsection{SWLI}


Surface roughness was measured on the Zygo NewView 200 Scanning White Light Interferometer (SWLI) (Zygo Corporation, Connecticut). A 10x objective lens was used for the SWLI analysis. Aged samples were aligned to ensure that the location of the roughness measurements coincided with those for the reflectance measurements.

\section{Results and Discussion}

\subsection{UV Aging Results}

The fluorescent and LED light panel applications of the coating under consideration require uniform reflection from all angles. Hence, diffuse reflectance, which spreads incident light uniformly across a range of angles, is more important than specular reflectance. Therefore, only diffuse reflectance is reported for this investigation. Change in diffuse reflectance during the coating's lifetime is a characteristic of its durability. Figure 1 shows diffuse reflectance measurements vs. wavelength obtained with the UV/Vis spectrophotometer for our coating before the aging process. An average of eight diffuse reflectance measurements obtained at eight different sampling points is plotted here. The reflectance abruptly drops near the UV region (below 400nm) due to significant absorption by $\mathrm{TiO}_{2}$ particles in that wavelength range [18]. Average reflectance measurements were carried out for samples subjected to UV-aging from 0 to 1000 hours. Figure 2 shows the effect of UV aging on diffuse reflectance at four different wavelengths ranging from 450 to $750 \mathrm{~nm}$. The diffuse reflectance generally decreases with aging time for all wavelengths, suggesting degradation.

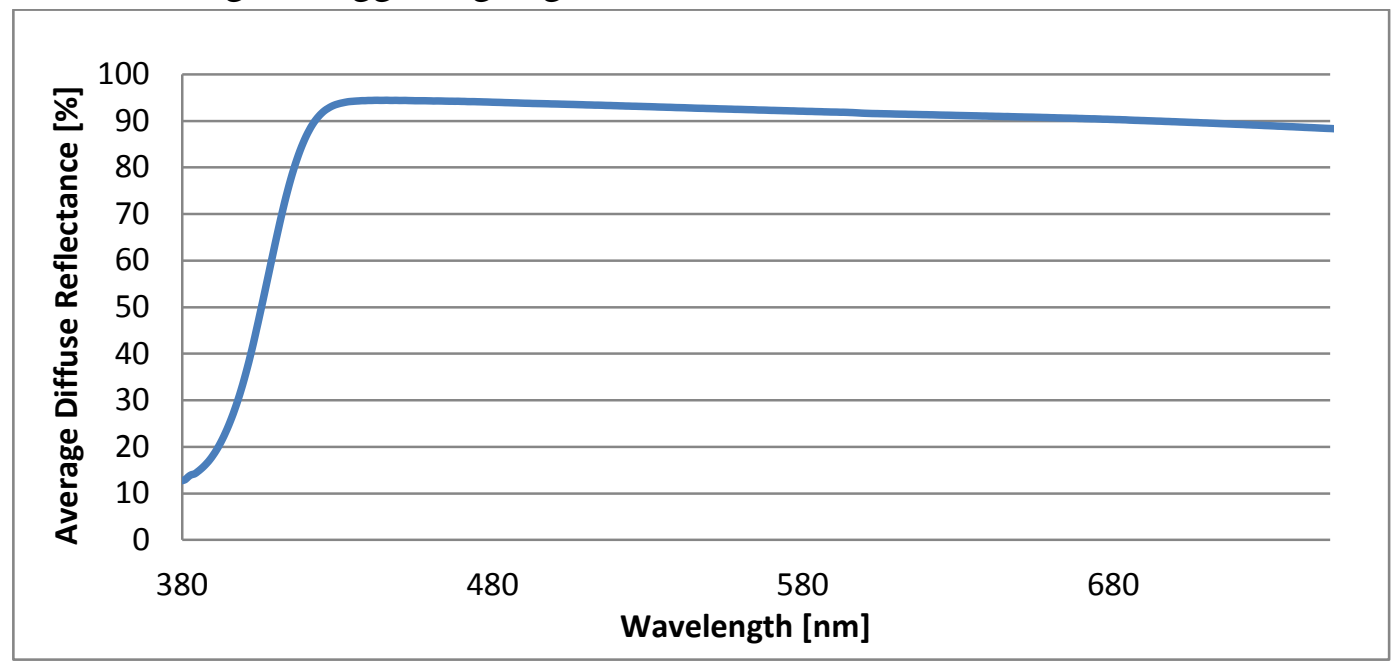

Figure 1 Diffuse reflectivity measurements vs. wavelength of the fresh coating 
In Figure 2, it should be noted that if the diffuse reflectance declines at the same rate over time for all wavelengths, i.e. the curves remain parallel as a function of aging, this will be observed as dulling. If the change is non-uniform across the wavelength range, the resulting effect will be observed as discoloration. Figure 2 is indicative of discoloration as the different wavelengths are affected differently over time. Furthermore, the overall reflectivity of the coating drops as a function of time. The polyurethane binder was expected to degrade due to the aggressive UV radiation. This degradation was investigated using ATR-FTIR.

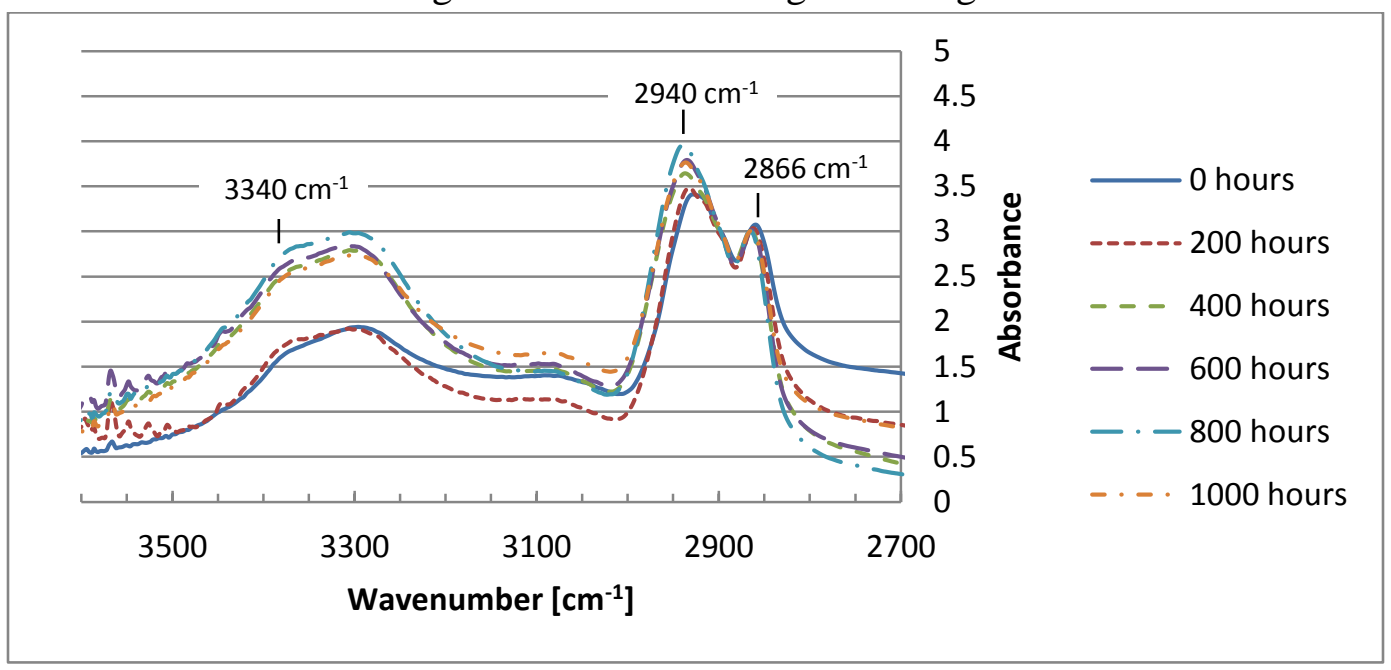

(a) 
(b)

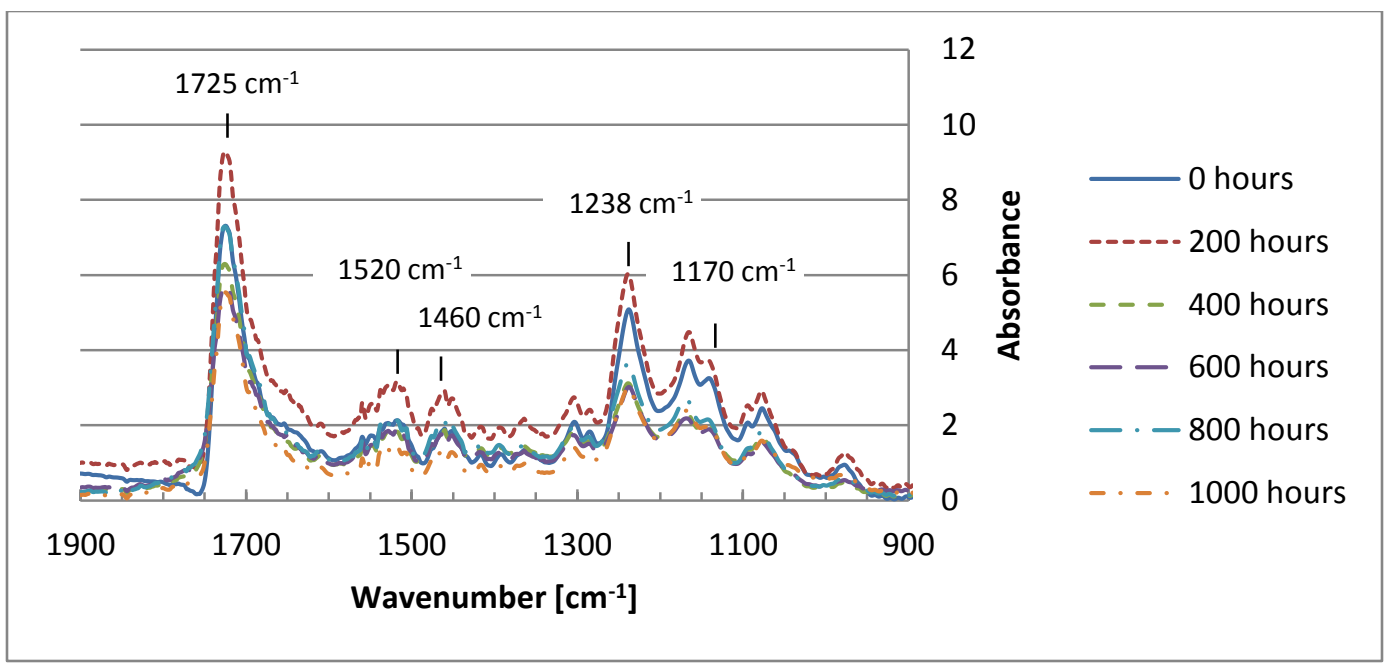

Figure 3 ATR-FTIR measurements in absorbance as a function of wave number is showing the effect of $\mathrm{UV}$ exposure at the interval of 200 hours on the relative magnitude of peak areas for the coatings formulated

Figure 3 shows the results of the ATR-FTIR analysis of the QUV aged samples. All spectra were baseline corrected and normalized at $2866 \mathrm{~cm}^{-1}$ [19]. To understand the chemical degradation mechanism caused by the QUV exposure, the ATR-FTIR analysis was conducted every 200 hours. An increase at $3370 \mathrm{~cm}^{-1}$ peak was observed after 400 hours of QUV exposure. This peak was associated with polyurea $(\mathrm{N}-\mathrm{H})$ stretching [19] and the increase in peak height indicates an increase in polyurea. The fluctuation at $2940 \mathrm{~cm}^{-1}$ was due to the fluctuation of the $\mathrm{CH}_{2}$ group. A reduction in peak height is observed at $1725 \mathrm{~cm}^{-1}$ due to QUV aging. This peak was attributed to the free $\mathrm{C}=\mathrm{O}$ in the polyurethane structure [20]. Furthermore, the $1520 \mathrm{~cm}^{-1}$ peak showed a decrease. This peak was attributed to the amide II -CO-NH- [21]. A steady decrease was observed at both $1460 \mathrm{~cm}^{-1}$ and $1238 \mathrm{~cm}^{-1}$. This suggests a $\mathrm{C}-\mathrm{H}$ and a $\mathrm{C}-\mathrm{O}$ stretching decrease [19]. Chain scission caused by QUV exposure is the reason for $\mathrm{C}-\mathrm{H}$ and $\mathrm{C}-\mathrm{O}$ decrease [19]. This formed carbamic acid, which further degrades due to its instability to carbon dioxide. This explains the decrease of $\mathrm{C}-\mathrm{O}$ and $\mathrm{C}-\mathrm{C}$ group evidenced by the decrease of C-O-C at $1238 \mathrm{~cm}^{-1}$ since the carbamic acid and carbon dioxide are only intermediate steps. Lastly, the decrease at $1238 \mathrm{~cm}^{-1}$ and $1170 \mathrm{~cm}^{-1}$ (-COO-) can be attributed to the overall film erosion effect [21].

From the ATR-FTIR data collected during the QUV aging process, it can be shown that QUV aging of the system causes UV induced photo-oxidations. For polyurethanes, it is widely acknowledged that UV induced photo-oxidation targets the methylene groups in the $\alpha$ position to the $\mathrm{N}-\mathrm{H}$ groups $[21,22]$. This can be seen in the chain scission, and in some cases reassociation, during the aging process. The results above agree with the degradation mechanism presented by both Yang and Kim [19, 20].

A secondary degradation mechanism has been hypothesized for this system under QUV aging. Though the UV radiation will directly impact and degrade the polymer binder as 

its properties. It has been widely reported that, in the presence of UV radiation $(<400 \mathrm{~nm})$, moisture, and air, $\mathrm{TiO}_{2}$ will create hydrogen peroxide $[23,24]$. It should be noted that, the UVB313 EL Fluorescent Lamp (Q-Lab Corporation, Ohio) used in the QUV aging has a peak radiation wavelength of $313 \mathrm{~nm}$, well below $400 \mathrm{~nm}$. Thus the $\mathrm{TiO}_{2}$ acts as a photocatalyst to encourage the production of hydrogen peroxide. The peroxide will accelerate the oxidation of the polymer binder, thus causing accelerated weathering. This can then cause the polymer binder and pigment to flake off or wash. The water-based polyurethane used in this study appears to undergo this degradation mechanism. Evidence of the breaking of double bonds in $\mathrm{C}=\mathrm{O}$ seen in the ATR-FTIR data at $1725 \mathrm{~cm}^{-1}$ can be attributed to the presence of hydrogen peroxide. Other evidence can be observed in the pitting found in the samples' surface as the aging progresses as shown in Figure 4. In a study conducted by Santos et al. [25], a combination of chalking and gloss reduction was observed in a similar polyurethane system exposed to UV radiation. Different polymers including polyurethanes were exposed to similar conditions while evaluating gloss reduction, color change, and chalking. Santos reported the polyurethane suffered 4-8 \% chalking in different UV aging conditions [26]. In a study conducted by Stachelek et al. [27], a phenol-based antioxidant was covalently attached to greatly reduce the rate of oxidation. It should be noted that the current system being tested did not include any oxidation inhibitors or antioxidants.

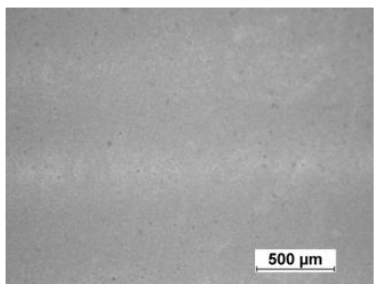

0 hours UV aged

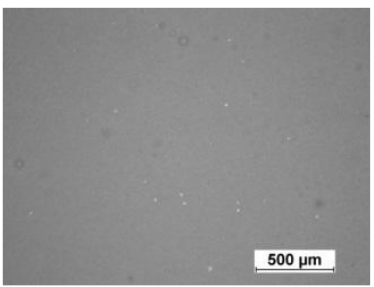

600 hours UV aged

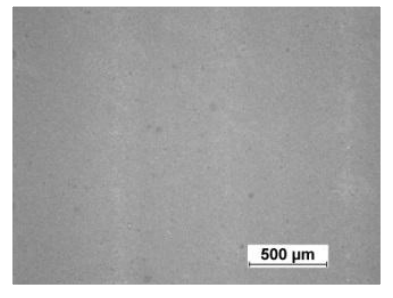

200 hours UV aged

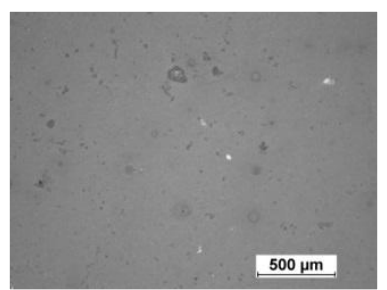

800 hours UV aged

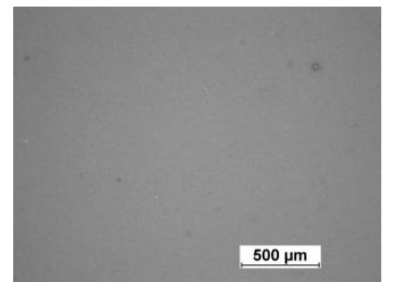

400 hours UV aged

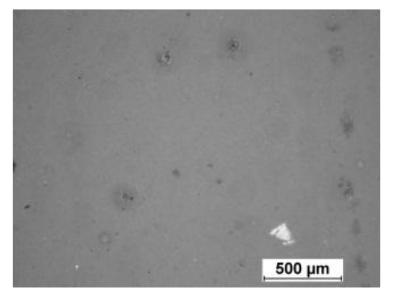

1000 hours UV aged

\section{Figure 4 Optical microscopy images of surface degradation during UV aging}

Figure 4 shows a clear trend in the surface change of the samples subjected to UV exposure as recorded by optical microscopy. With increasing exposure, defects on the surface of the samples grow in size and number. It should be noted that although the effects of UV were present throughout the aging process, it was only after 400 to 600 hours that the surface defects started to affect the diffuse reflectance. From the trend in the optical microcopy images, a possible mechanism for the degradation of diffuse reflectance can be proposed. Through the 
process of UV polymer degradation and chalking, the surface initially becomes more uniform. This affects the diffuse reflectance minimally. Further degradation from the UV-induced photooxidation and chalking causes defects to form, first observed mildly at 400 hours and more prominently around 600 hours of aging. When comparing with the reflectance measurements in Figure 2, one observes the start of the decrease in reflectance at 400 to 600 hours of UV irradiation, which continues to the end of the aging period.

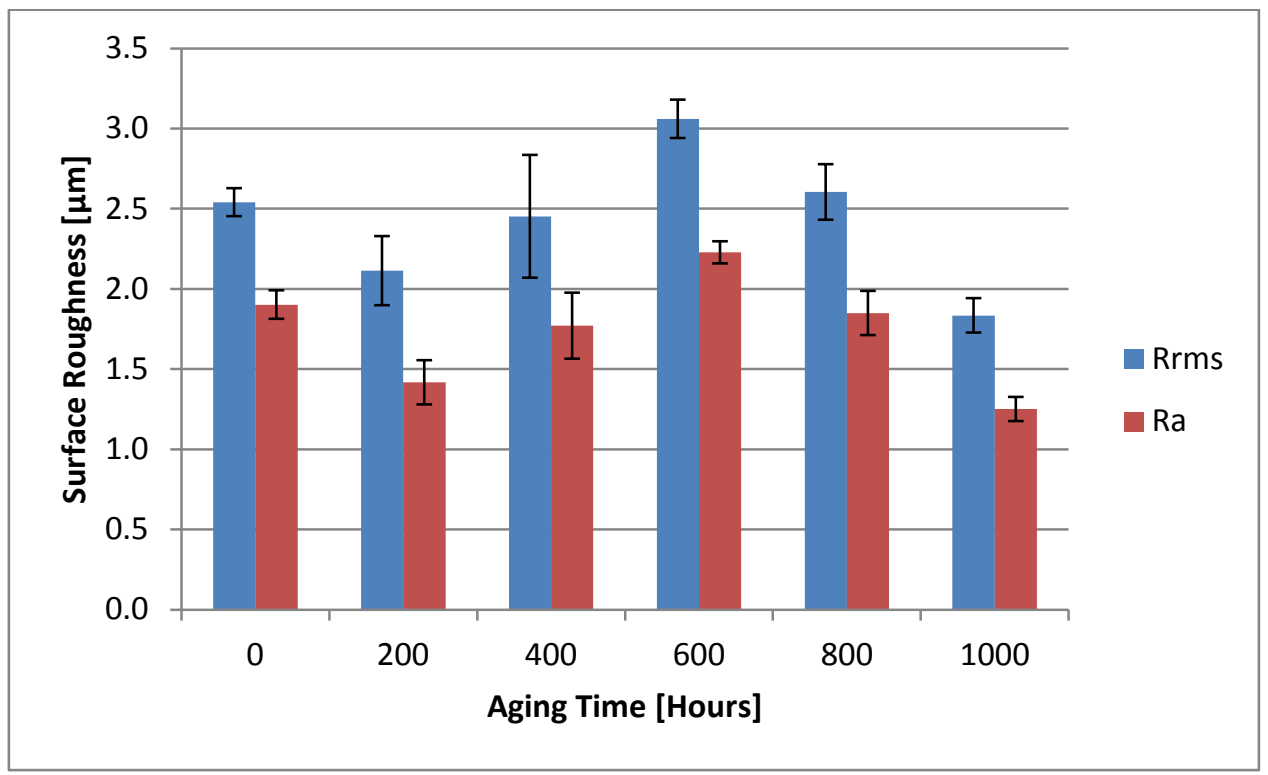

Figure 5 Surface roughness measurements during UV aging by SWLI

At low wavelengths, the trends for the diffuse reflectance in Figure 2 seem to follow the surface roughness measurements shown in Figure 5. It should be noted that $R_{a}$ is the arithmetic average of the absolute values of the surface roughness while the $\mathrm{R}_{\mathrm{rms}}$ is the root mean square of the roughness measurements. The chemical degradation caused by the UV radiation and the consequent chalking changes the surface roughness. While SWLI measures the height differences in the system, the areal size of the roughness is not quantified. It is proposed that the decrease in reflectance at high wavelengths is correlated to the presence of the larger defects observed with the optical microscope. However, the optical microscope cannot capture the smaller defects, which could affect the reflectance at lower wavelengths due to magnification and contrast limitations. Since SWLI is sensitive to these small pores, it is likely that SWLI is depicting this trend more clearly.

\subsection{Water Immersion Aging Results}




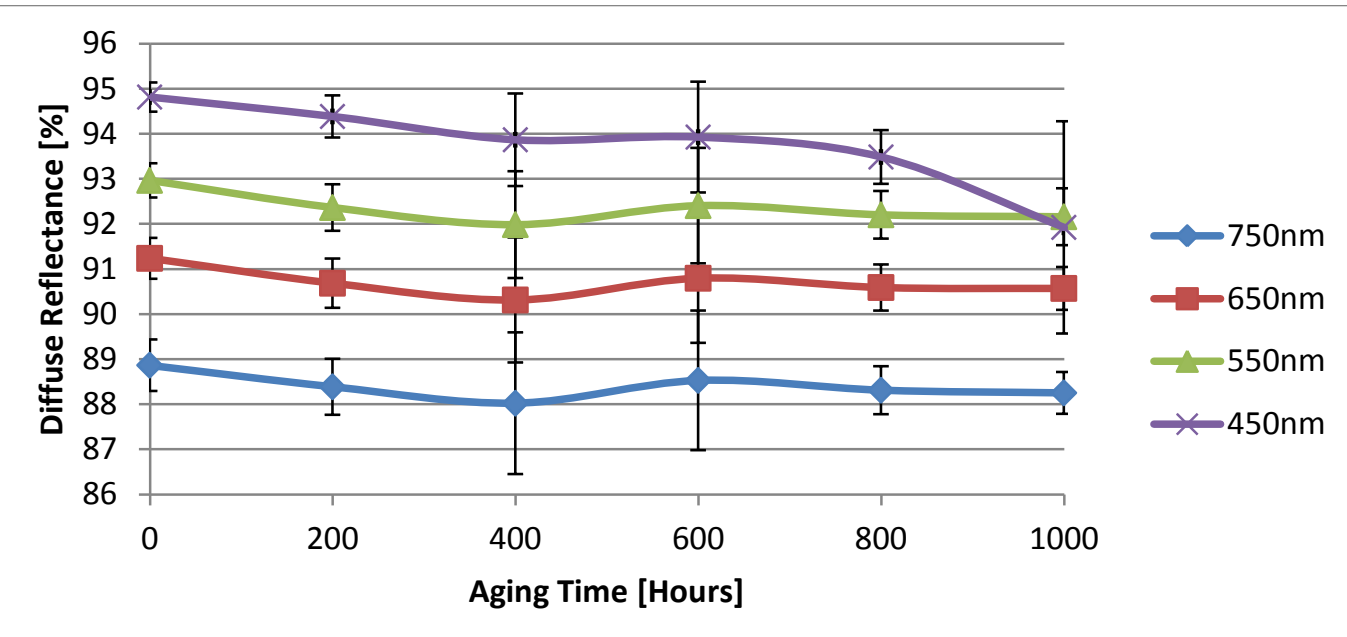

Figure 6 Diffuse reflectivity measurements vs. water immersion time for four different wavelengths.

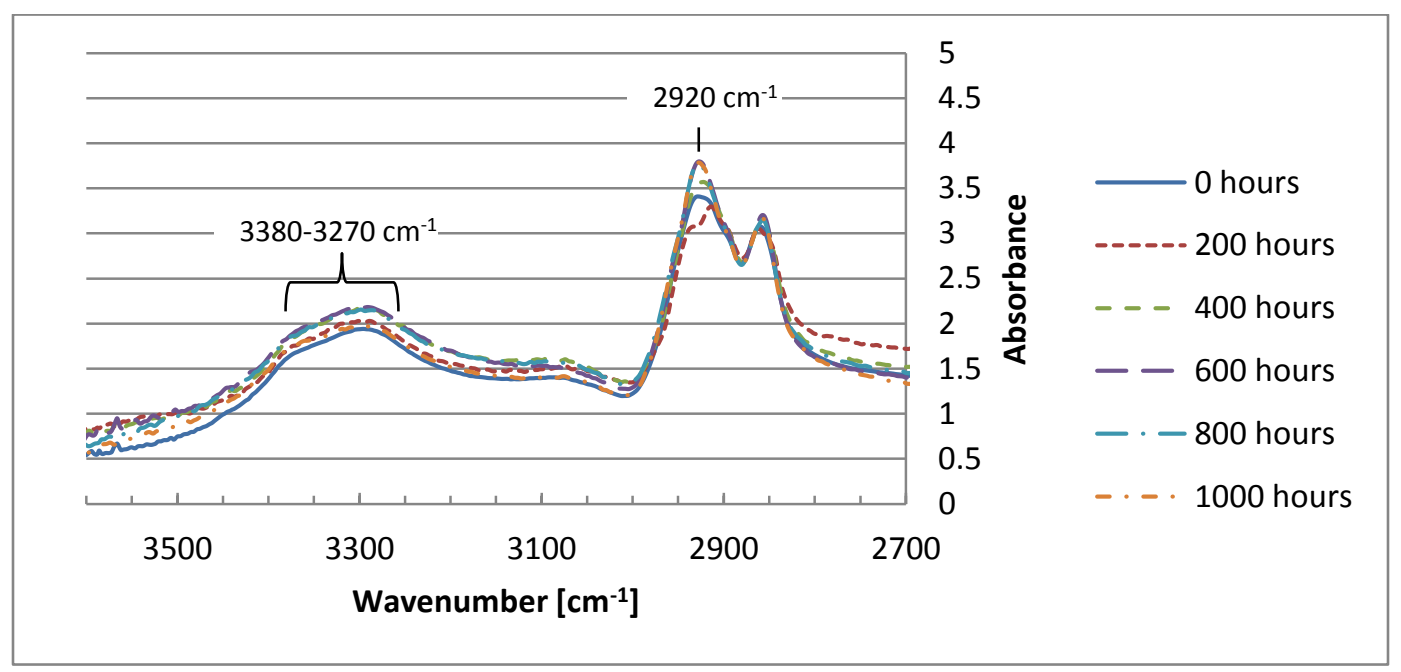

(a)

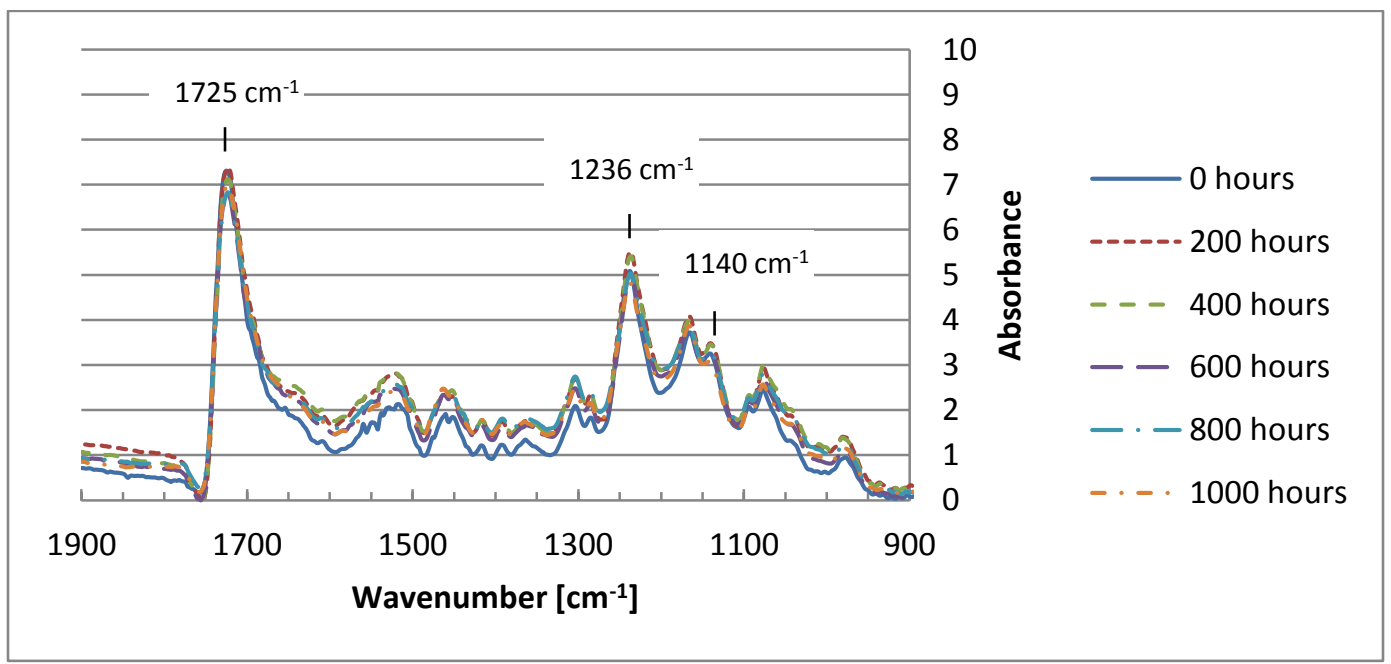


(b)

Figure 7 ATR-FTIR measurements in absorbance as a function of wave number is showing the effect of water immersion at the interval of 200 hours on the relative magnitude of peak areas for the dry coatings

Similarly to the QUV aging, water immersion samples were aged for 1000 hours and tested at 200-hour increments. Figure 6 shows the change in diffuse reflectance as a function of water immersion time. A slight dulling can be observed in the initial 200 hours with minimal color change. Discoloration becomes apparent only after 800 hours. Between 800 hours and 1000 hours, the sample had become noticeably yellow, indicating a higher absorbance in the blue range. Figure 7 shows the ATR-FTIR spectra for the water aged samples. In this figure, we can observe the slight increase then decrease of the 3380-3270 $\mathrm{cm}^{-1}$ wavelength range [19]. This peak is responsible for the polyurea N-H bond. Furthermore, the peak at $2920 \mathrm{~cm}^{-1}$ correlating to $\mathrm{C}-\mathrm{H}_{2}$ shows an increase. The peak at $1725 \mathrm{~cm}^{-1}$ shows a decrease as well suggesting a decrease in the free carbonyl [20]. Peaks at $1236 \mathrm{~cm}^{-1}$ and $1140 \mathrm{~cm}^{-1}$ correlating to CO and COC bonds also show a decrease over time. This suggests that these bonds are being broken by chain scission [28]. There may be reassociation seen with the increase in $\mathrm{NH}$ of the polyurea, however, that is further degraded after 800 hours.

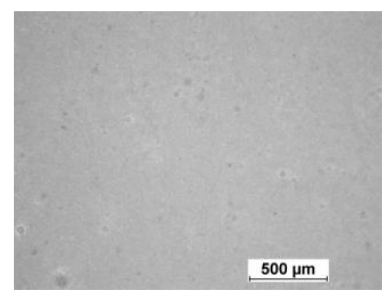

0 hour water aged

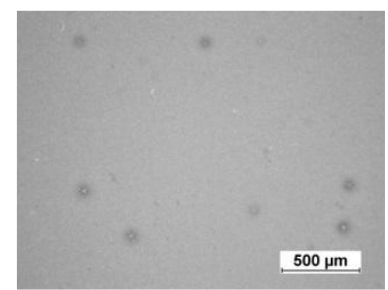

600 hours water aged

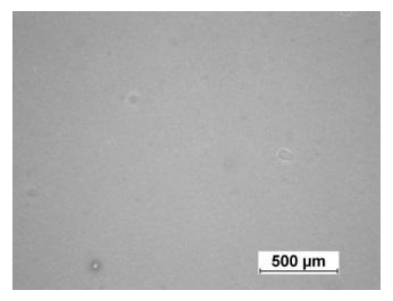

200 hours water aged

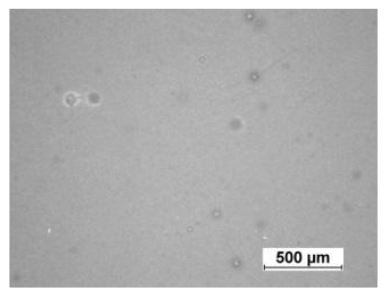

800 hours water aged

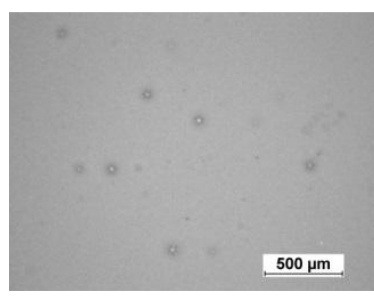

400 hours water aged

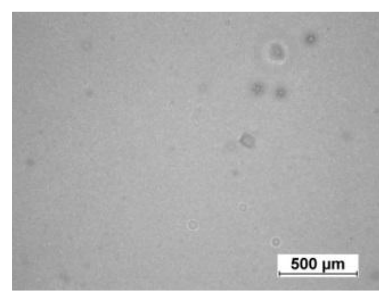

1000 hours water aged

Figure 8 Optical microscopy images of surface degradation during water immersion aging 

aging caused by the penetration of water and subsequent blistering. Blistering is not uncommon especially in polyurethane systems. Although the samples were patted dry prior to the reflectivity measurements, not all of the water could be removed. Also, the blisters formed by water penetration do not self-heal when dried since the system is partially cross-linked. These effects would contribute to lowering the overall reflectance. Aside from the blistering discussed earlier, the formation of holes is observed in the coating between 200 to 400 hours in Figure 8 . Thus the microscopy images appear to confirm that physical defects are the likely cause for the slight decrease in reflectance. After this point in the aging, both the microscopy and reflectance data suggest that most of the defects have formed and minimal changes in reflectance are observed for the high wavelength regions of the visible spectrum. The reflectance at $450 \mathrm{~nm}$ seen in Figure 6 shows a close correlation to both the $R_{a}$ and $R_{r m s}$ from the SWLI data in Figure 9. This observation is similar to that of the UV aging samples. This

\subsection{Thermal Aging Results}




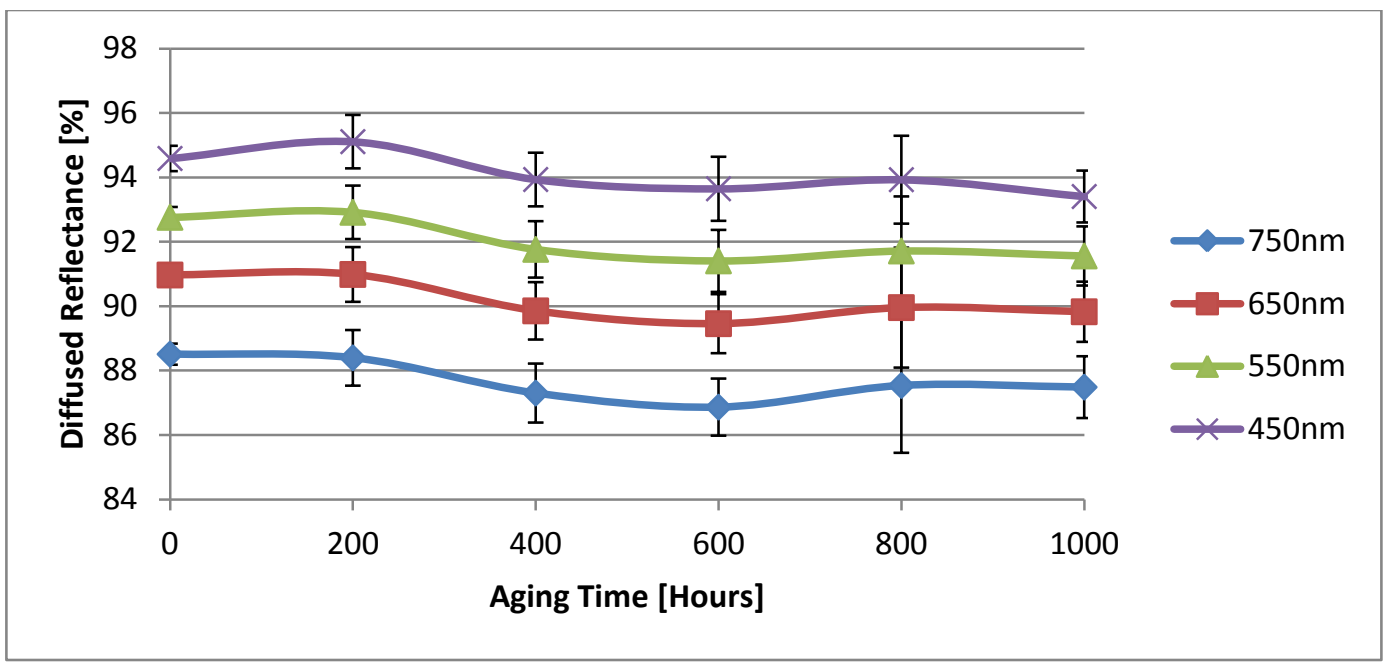

\section{Figure 10 Diffuse reflectivity measurements vs. thermal aging time for four different wavelengths.}

The reflectance data from Figure 10 show minor fluctuations that ultimately reduce the overall reflectance. Figure 11 shows the chemical changes occurring during the thermal aging. Minor changes are observed in the 3380 to $3250 \mathrm{~cm}^{-1}$ range. The largest changes can be observed at $2925 \mathrm{~cm}^{-1}, 1725 \mathrm{~cm}^{-1}, 1236 \mathrm{~cm}^{-1}$ and $1170 \mathrm{~cm}^{-1} .2925 \mathrm{~cm}^{-1}$ corresponds to the fluctuation of the $\mathrm{CH}_{2}$ in the system while the decrease at $1725 \mathrm{~cm}^{-1}$ again corresponds to the decrease in free carbonyl in the polyurethane binder [20]. Zhang [21] refers to the decrease at $1236 \mathrm{~cm}^{-1}$ and $1170 \mathrm{~cm}^{-1}$ as resulting from the overall film erosion. Similar to water immersion aging, optical microscopy indicates the formation of defects as the material is exposed to thermal cycling. This formation of defects can be attributed to physical aging caused by a mismatch in the coefficient of thermal expansion (CTE) between the binder, pigment, and substrate as listed in Table 3. As the temperature changes, the mismatch in thermal expansion of each component causes internal stresses, which are released by the formation of pores and cracks.

Table 3 CTE of Components

\begin{tabular}{cc}
\hline Component & CTE Values $\left(\mathbf{1 0}^{-\mathbf{6}} \mathbf{m} / \mathbf{m}-\mathbf{K}\right)$ \\
\hline Polyurethane Binder & $70-150[29]$ \\
$\mathrm{TiO}_{2}$ Pigment & $8.9-9.4[30,31]$ \\
Aluminum Substrate & $19.2-24.6[32]$ \\
\hline
\end{tabular}

Figure 12 indicates that temperature cycling has caused the formation of defects due to thermal stresses. Small defects form throughout the aging process. The adverse effects of the defects can be seen in the reflectivity measurements. The downward trend in reflectivity is well correlated to the growth in defects. The reflectance at the low wavelength in Figure 10 shows some resemblance to the trend observed in the surface roughness measured by SWLI presented 
in Figure 13. In this case, there seems to be an outlier at 200 hours observed by the uncharacteristically large deviation.

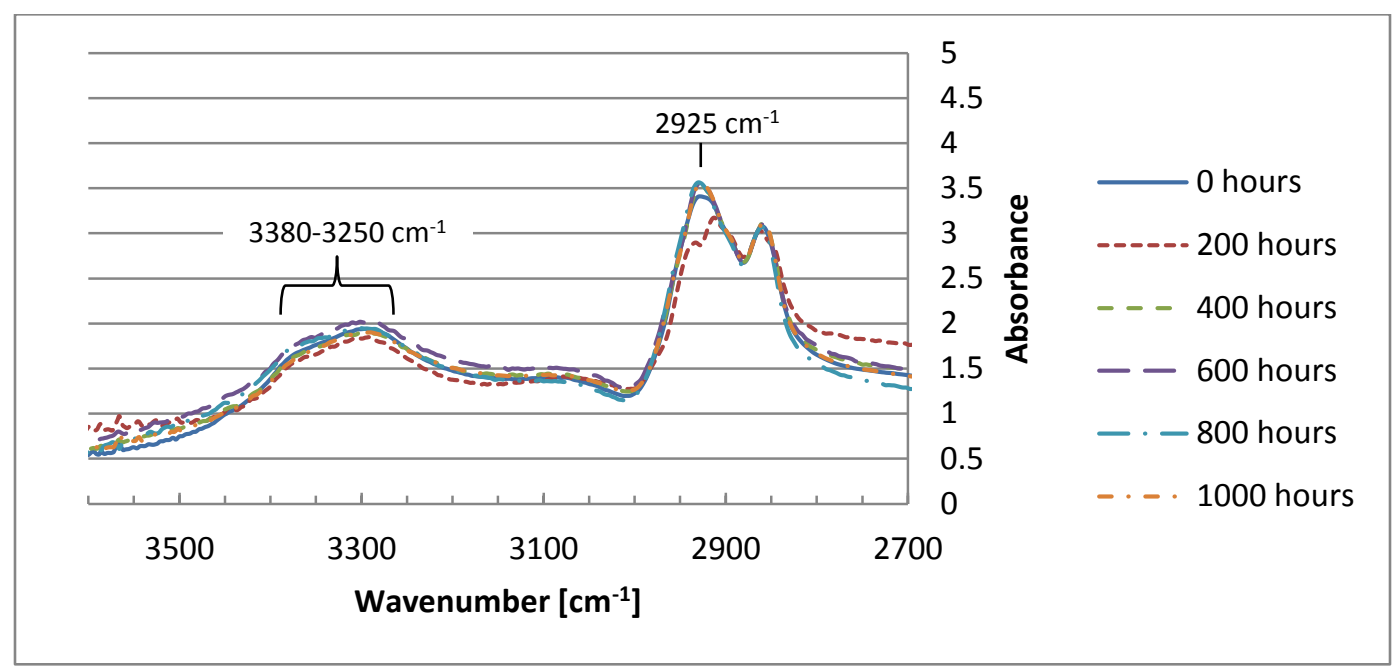

(a)

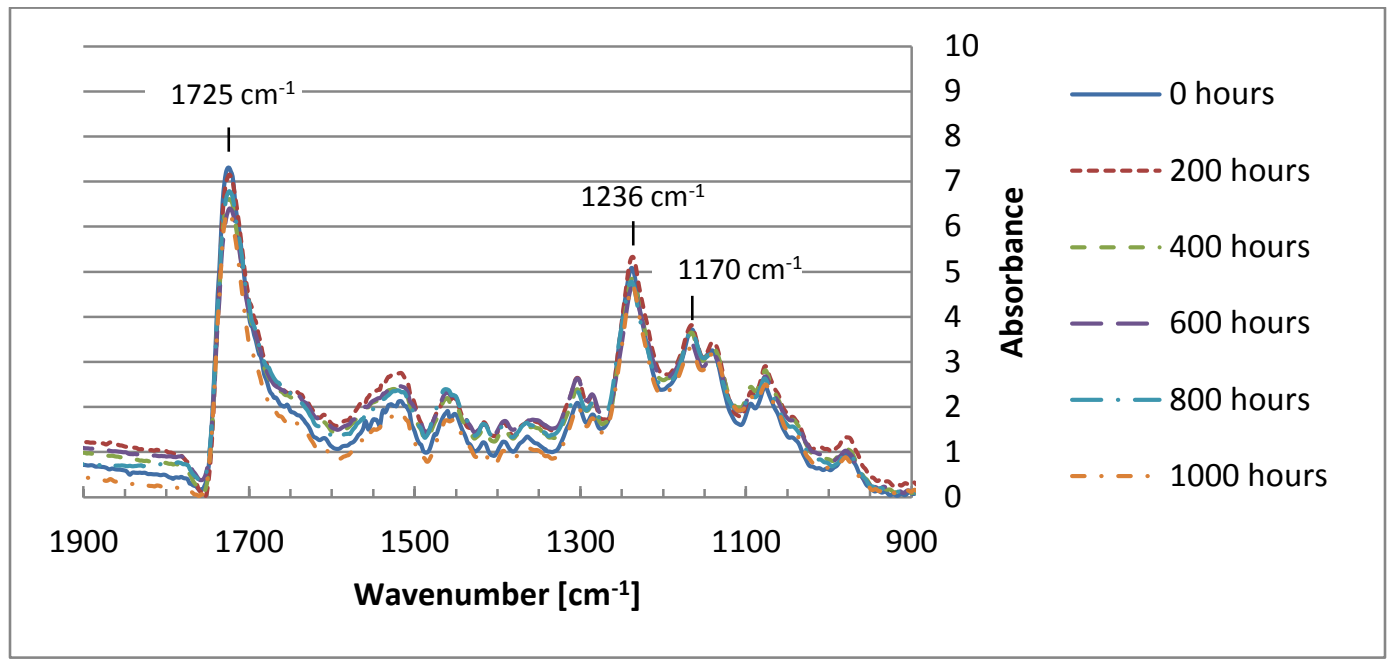

(b)

Figure 11 ATR-FTIR measurements in absorbance as a function of wave number is showing the effect of thermal aging at the interval of 200 hours on the relative magnitude of peak areas for the dry coatings 


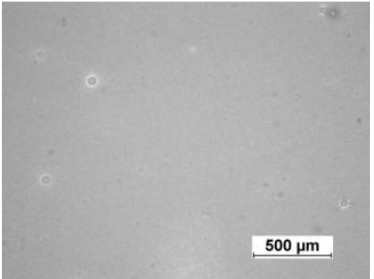

0 hours thermal aged

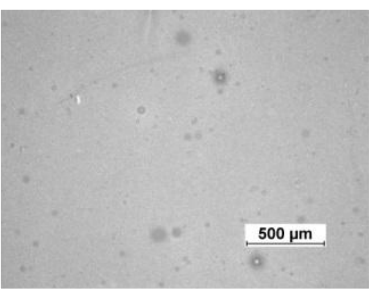

600 hours thermal aged

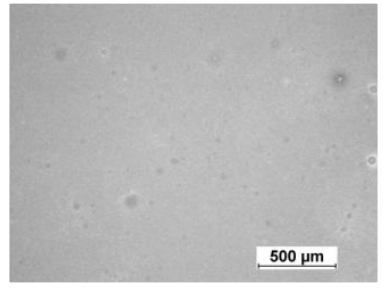

200 hours thermal aged

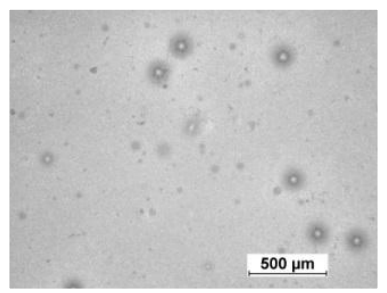

800 hours thermal aged

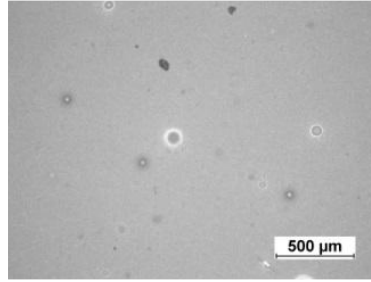

400 hours thermal aged

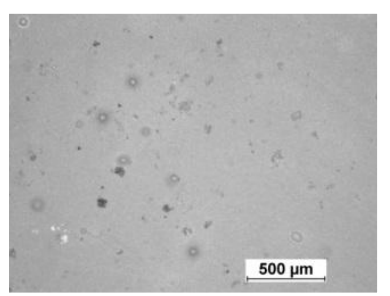

1000 hours thermal aged

Figure 12 Optical microscopy images of surface degradation during thermal aging

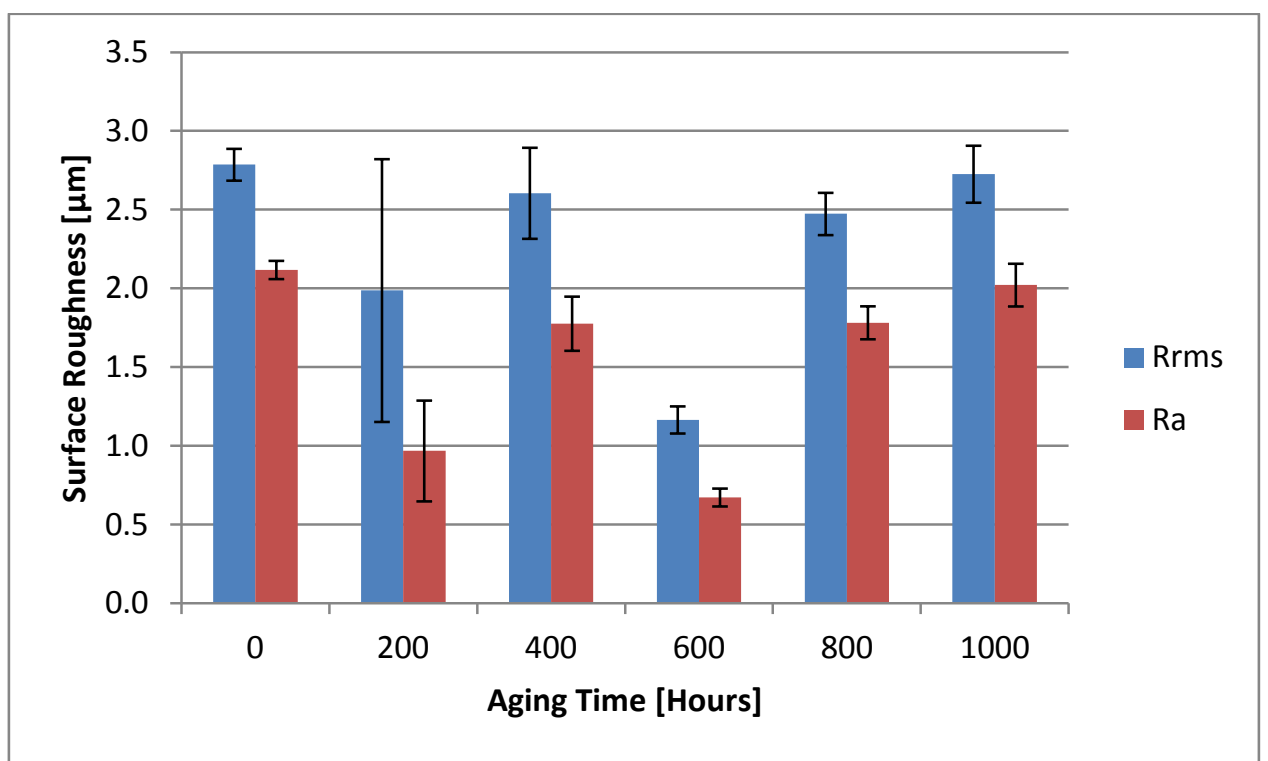

Figure 13 Surface roughness measurements during thermal aging by SWLI

\section{Conclusions}

A water-based polyurethane coating was aged in three different ways to determine the durability of its reflective properties, and to understand the underlying degradation mechanisms. The three methods of aging consisted of UV irradiation, water immersion, and thermal cycling over a period of 1000 hours. Exposure to these aging environments affected the coating in both physical and chemical ways causing a complex degradation of the system and its reflective properties.

UV irradiation causes both a direct degradation of the polymer binder, as well as the secondary degradation mechanism of chalking. Although both are caused by the high intensity of UV radiation, the actual mechanisms differ greatly. In the first case, UV radiation directly breaks 

susceptible to breaking as they tend to absorb more energy at smaller wavelengths such as UV. The second degradation mechanism is caused by the pigment absorbing UV radiation in the presence of humidity and air leading to the formation of hydroxyl radicals, which then react with other radicals to form hydrogen peroxide. Hydrogen peroxide reacts to break double bonds in both the original polymer binder and intermediate compounds to further degrade the binder. Chemical degradation causes physical effects such as chalking and pitting which directly reduce the diffuse reflectivity. The greater the number of defects in the coating, the lower the reflectance since incident light is either unable to escape the defect or is absorbed by the substrate. It is noteworthy that the surface roughness trend seems to correlate well with that of the reflectance at small wavelengths, while the surface defects observed using the optical microscope correlate well at higher wavelengths.

Water immersion aging causes physical rather than chemical degradation of the coating. Only small chemical changes were observed towards the end of the aging process, whereas the greatest impact on the coating's durability is water penetration. Water absorption into the coating causes blistering. When dried, this leads to void formation, and in some cases, retention of residual water within the coating. These voids decrease reflectance. Furthermore, the blistering process ends within the first 200 to 400 hours of exposure, and the performance loss is permanent. Similar to UV aging, the surface roughness trend from SWLI closely followed that of the reflectance drop at small wavelengths.

Thermal cycling causes minimal chemical change similar to water immersion. Furthermore, physical aging is the primary cause of performance loss. Thermal stresses resulting from a mismatch in the coefficients of thermal expansion of the binder, pigment and substrate cause defects to form. The larger the defects, the greater the reflectance drop.

Though much of the degradation observed seems to be similar, the different sources of aging can be seen at different time of the aging process. UV aging shows holes and defects form between 400 and 600 hours with reflectance drops around 400 hours of exposure. Water aging shows the formation of holes and defects between 200 and 400 hours and the reflectance decreases immediately. Thermal aging shows signs of defects starting at 200 hours and continues to form. Reflection drops between 200 and 400 hours. To determine the dominant aging mechanism, one can look to the timing of the reflectance drops and formation of the hole and defect formation. Furthermore, one can determine the dominant degradation through chemical analysis through FTIR.

Overall, aging by exposure to QUV, water immersion, or thermal cycling successfully demonstrated performance degradation through chemical and physical aging. Studying each aging mode in isolation provided insight into the underlying mechanisms and will allow for proper approaches to reduce the effects of the aging for the desired application. Further study is required to confirm the correlation between the surface roughness as measured by SWLI and the reflectivity at small wavelengths. Regardless, surface examination for the formation of defects has proved to be a reliable indicator for the degradation in diffuse reflectance. 


\section{Acknowledgments}

The authors acknowledge support for this work from U. S. Department of Energy and discussions with Eric Teather, President of WhiteOptics LLC. The authors are grateful to Professor S. Ismat Shah, Department of Materials Science and Engineering, University of Delaware, for providing the use of the UV/Vis spectrophotometer.

\section{References}

1. L. M. Gartland, Cool coatings heat up savings, Maintenance Solutions, January 1999.

2. R. Tatum, Cool roofs, hot topic, Building Operating Management, May 1999.

3. Environmental Protection Agency, 40 CFR Parts 59, 1998.

4. M. Szycher, Szycher's handbook of polyurethanes, CRC Press, 1999.

5. W. Blank, Progress in Organic Coatings 20 (1992) 235-259

6. A. Ludwick, H. Aglan, M. Abdalla, M. Calhoun, Journal of Applied Polymer Science 110 (2008) 712-718

7. C. Decker, F. Masson, R. Schwalm, Polymer Degradation and Stability 83 (2004) 309-320

8. L. Wang, G. Liang, G. Dang, F. Wang, X. Fan, W. Fu, Chinese Journal of Chemistry 23 (2005) 1257-1263

9. R.N. Jana, H. Bhunia, High Performance Polymers 22 (2010) 3-15

10. S. Saha, D. Kocaefe, Y. Boluk, A. Pichette, Progress in Organic Coatings 70 (2011) 376382

11. E. Teather, Y. Xiaorong, (2014) U.S. Patent No. 8,734,940 B2, U.S. Patent and Trademark Office

12. D. Stoye, W. Freitag, Paints, coatings, and solvents, Wiley-VCH, 1998, 161.

13. S. Farrokhpay, Adv. Colloid Interface Sci. 151 (2009) 24-32.

14. J. Clayton, Surface Coatings International 9 (1997) 414-420.

15. ASTM standard D 4674, Standard practice for accelerated testing for color stability of plastics exposed to indoor office environments, Annual Book of ASTM Standard

16. ASTM standard D 870-02, Standard practice for testing water resistance of coatings using water immersion, Annual Book of ASTM Standard

17. ASTM standard D 6944-03, Standard test method for resistance for cured coatings to thermal cycling, Annual Book of ASTM Standard

18. Ti-Pure® Titanium Dioxide for Coatings, DuPont Titanium Technologies Literature, USA, 2007, 10.

19. X.F. Yang, C. Vang, D.E. Tallman, G.P. Bierwagen, S.G. Croll, S. Rohlik, Polymer Degradation and Stability 74 (2001) 341-351

20. H. Kim, M.W. Urban, Langmuir 16 (2000) 5382-5390

21. Y. Zhang, J. Maxted, A. Barber, C. Lowe, R. Smith, Polymer Degradation and Stability 98 (2013) 527-534

22. L. Irusta, M. J. Fernandex-Berridi, Polymer Degradation and Stability 63 (1999) 113-119 
23. S. Pappas, R. Fischer, Journal of Paint Technology 46 (1974) 65-72

24. R, Gerteis, A. Elm, Journal of Paint Technology 43 (1971) 99-106

25. D. Santos, C. Brites, M.R. Costa, M.T. Santos Progress in Organic Coatings 54 (2005) 344352.

26. D. Santos, A. Vieira, E. Almeida, Materials and Corrosion 51 (2000) 481-485.

27. S. Stachelek, I. Alferiev, H. Choi, C. Chan, B. Zubiate, M. Sacks, R. Composto, I, Chen, R. Levy, J. Biomed. Mater. Res. 78A (2006) 653-661

28. A. Boubaki, K. Elleuch, N. Guermazi, H. F. Ayedi, Materials and Design 30 (2009) 39583965

29. K. Morimoto, T. Suzuki, R. Yosomiya, Polymer Engineering and Science 24 (1984) 943949

30. K.V. Rao, S.V.N. Naidu, L. Iyengar, Journal of the American Ceramic Society 53 (1970) $124-126$

31. D.R. Hummer, P.J. Heaney, J.E. Post, Powder Diffraction 22 (2007) 352-357

32. E.E. McCollough, Journal of General and Applied Physics 1 (1931) 334-339 